EPJ Web of Conferences 41, 07018 (2013)

DOI: $10.1051 /$ epjconf/20134107018

(C) Owned by the authors, published by EDP Sciences, 2013

\title{
Probing how initial retinal configuration controls photochemical dynamics in retinal proteins
}

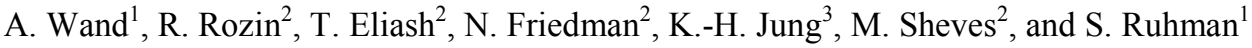 \\ ${ }^{1}$ Institute of Chemistry, The Hebrew University of Jerusalem, Jerusalem 91904, Israel \\ ${ }^{2}$ Department of Organic Chemistry, The Weizmann Institute of Science, Rehovot 76100 , Israel \\ ${ }^{3}$ Department of Life Science, Sogang University, Seoul 121-742, South Korea
}

\begin{abstract}
The effects of the initial retinal configuration and the active isomerization coordinate on the photochemistry of retinal proteins (RPs) are assessed by comparing photochemical dynamics of two stable retinal ground state configurations (all-trans, 15anti vs. 13-cis,15-syn), within two RPs: Bacteriorhodopsin (BR) and Anabaena Sensory Rhodopsin (ASR). Hyperspectral pump-probe spectroscopy shows that photochemistry starting from 13-cis retinal in both proteins is 3-10 times faster than when started in the all-trans state, suggesting that the hastening is ubiquitous to microbial RPs, regardless of their different biological functions and origin. This may also relate to the known disparity of photochemical rates between microbial RPs and visual pigments. Importance and possible underlying mechanisms are discussed as well.
\end{abstract}

\section{Introduction and Motivation}

Photochemistry in retinal proteins is determined both by properties of the retinal chromophore and by its interactions with the surrounding protein [1]. The initial retinal configuration, and the active isomerization coordinates, must play an important role in that respect, as illustrated by the striking differences between the photoisomerization dynamics in visual pigments which start 11-cis, and those in microbial retinal proteins (MRPs) which start all-trans. While absorption of light in the former unleashes ballistic sub-picosecond internal conversion (IC) [2], MRPs re-access the ground state roughly ten times slower, possibly via potential barrier crossing.

The importance of the initial retinal configuration can be best assessed in isolation by comparing photoisomerization dynamics of different retinal ground state configurations within the same protein. This is achievable in Bacteriorhodopsin (BR) and Anabaena Sensory Rhodopsin (ASR), two MRPs which exist in two stable ground state forms of their retinal protonated Schiff base chromophore: alltrans,15-anti $\left(\mathrm{X}_{\mathrm{AT}}\right)$ and 13-cis,15-syn $\left(\mathrm{X}_{13 \mathrm{C}}\right)$. Experiments reported here compare IC dynamics starting from either ground state by conducting hyperspectral pump-probe spectroscopy with sub100 fs temporal resolution on dark-adapted and light-adapted samples of both proteins [see 3,4].

\section{Main Results}

A home-built multipass amplified Ti:Sapph apparatus was used to seed a TOPAS (pump) and to generate supercontinuum white light probe pulses from a sapphire plate. The former was compressed

This is an Open Access article distributed under the terms of the Creative Commons Attribution License 2.0, which permits unrestricted use, distribution, and reproduction in any medium, provided the original work is properly cited. 
to $\sim 25 \mathrm{fs}$ in a slightly misaligned zero-dispersion grating shaper, and dispersion of the latter was corrected by a time-correction procedure: signal interpolation and time-axis shifting [4]. Darkadapted and fully light-adapted BR and ASR samples, whose retinal isomeric compositions were determined by HPLC measurements, were compared.
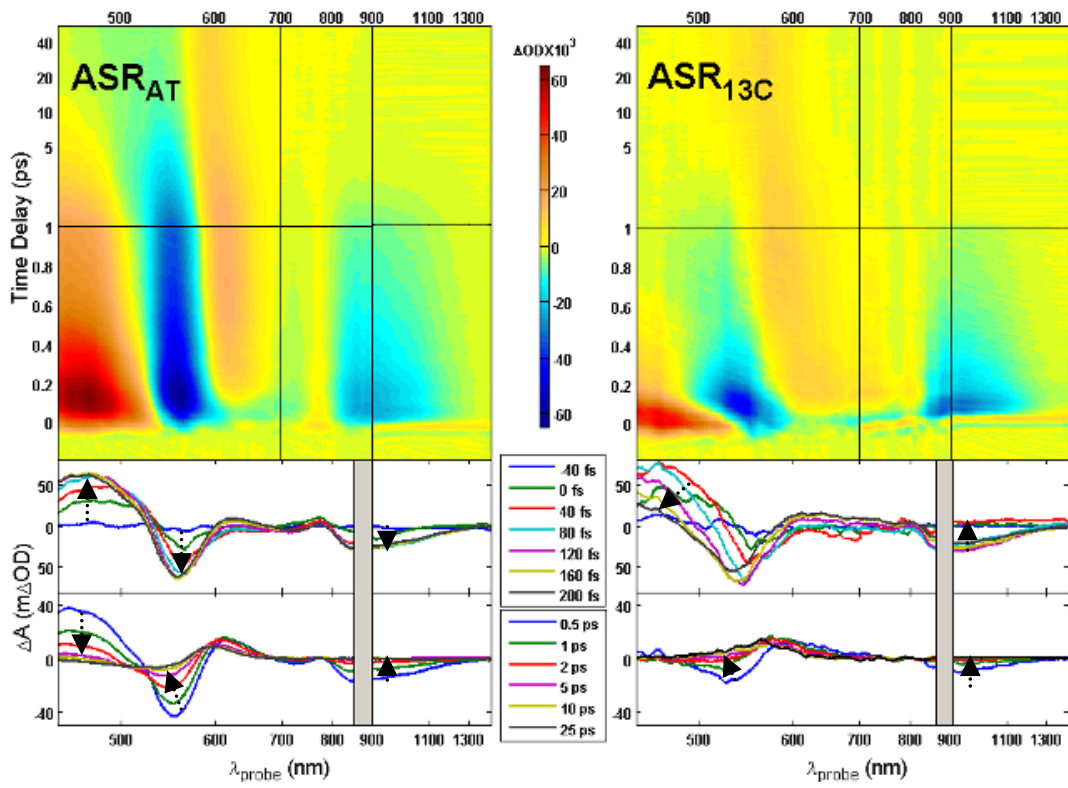

Fig. 1. Transient hyperspectral difference absorption spectra of $\operatorname{ASR}_{\mathrm{AT}}$ (left) and $\mathrm{ASR}_{13 \mathrm{C}}$ (right), shown as contour maps (upper panels). Lower panels depict sequences of temporal cuts of the same data at the designated pump-probe delays (arrows pointing at the temporal evolution direction).

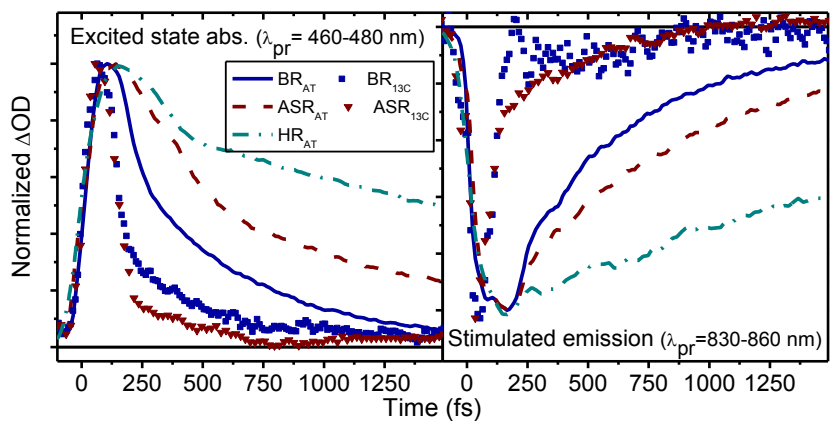

Fig. 2. Excited state absorption (left panel) and stimulated emission (right panel) signals for $B_{A T}$, $\mathrm{BR}_{13 \mathrm{C}}, \mathrm{ASR}_{\mathrm{AT}}, \mathrm{ASR}_{13 \mathrm{C}}$ (from this study), and $\mathrm{HR}_{\mathrm{AT}}$ (taken from Ref [5]), demonstrating shortening of the excited state lifetimes of the 13-cis configurations compared to the all-trans ones.

Figure 1 presents the transient absorption of ASR in the initial $\sim 50$ ps following photoexcitation for $\mathrm{ASR}_{\mathrm{AT}}$ (left) and $\mathrm{ASR}_{13 \mathrm{C}}$ (right). The latter is derived by a properly weighted subtraction of the recorded light-adapted and dark-adapted datasets (see Ref [4] for details). Starting with the former, it exhibits characteristics which are reminiscent of similar data recorded for other MRPs: (a) rapid buildup of excited state absorption and emission to the blue and to the red of the $\sim 550 \mathrm{~nm}$ ground state bleach, respectively. (b) sub-ps spectral shifting of these bands. (c) multiexponential IC within a few ps, leaving behind the signature of the red-shifted ground state product " $\mathrm{K}$ ". A totally different mapping is observed in the case of $\mathrm{ASR}_{13 \mathrm{C}}$, demonstrating a $\sim 10$-fold 
enhancement of IC rates (equivalent to a lifetime of ca. 200-300 fs), manifested by a continuous band-shifting and a sub-ps buildup of an isomerized (all-trans, 15-syn) "K" photoproduct signature. The results obtained for the case of $\mathrm{BR}_{13 \mathrm{C}}$ vs. $\mathrm{BR}_{\mathrm{AT}}$, not shown here (see ref 3), demonstrate the same trends as well, though to a lesser degree of acceleration, contradicting the findings of the pioneering work of Pertich et al. who concluded that both isomers share similar kinetics [6]. Figure 2 demonstrates the hastening of the IC in the 13-cis ground state of both proteins, whose excited state decay borders on our experimental time resolution, while the photoexcited all-trans proteins emit, and absorb, well beyond a picosecond, as does Halorhodopsin (HR) as well. The all-trans forms also include an initial spectral stagnation period on the order of a few hundreds of femtoseconds.

\section{Discussion and Summary}

BR, the well known proton pump, and the newly-identified photoswitchable ASR [7] perform very different biological functions. Nonetheless, both exhibit much faster timescales of IC when initiated in the 13-cis rather than all-trans ground state of the retinal, thus suggesting that the disparity in rates may be ubiquitous to all MRPs. This may also bear on the well known enhancement in photoisomerization rates going to the visual pigments, as the latter also start the course of photoreception in a cis retinal configuration, in that case 11-cis.

A few tentative explanations might be given to rationalize our observations based on existing knowledge. One possibility is the existence of a barrier en route to the conical intersection in the alltrans coordinate, which is absent in the 13-cis one. Even a small barrier, such as that which might have been recorded in a recent QM/MM study of bidirectional reaction coordinates in ASR [8], can account for an order of magnitude differences in IC rates and for multi-exponential kinetics [9]. A second explanation relates to the steric structure of the retinal chromophore within the protein pocket rather that to excited state topography. While pre-twisting and crowding are readily used to substantiate the ballistic dynamics of the ("sprung") 11-cis retinal in the visual pigment, these factors were generally overlooked in structural studies of all-trans vs. 13-cis MRPs, for which the ground state isomers are usually described as nearly planar. A closer look, however, suggests that the $\mathrm{C}_{14}{ }^{-}$ $\mathrm{C}_{15}$ single bond - directly next to the photoisomerizing $\mathrm{C}_{13}=\mathrm{C}_{14}$ bond - is considerably twisted in the 13-cis isomer only. Upon photon absorption, and due to the known bond order alternation nature of the excited state, this might give more impetus for the $\mathrm{C}_{13}=\mathrm{C}_{14}$ isomerization, supplying us with the "spring" sought for here, as was suggested by Garavelli and co-workers for Rhodopsin and model systems [2], and interestingly similar to the effects observed in artificial molecular switches [10].

To conclude, the results show that retinal IC in MRPs can take place as swiftly as in visual pigments when the retinal is in the 13-cis stable ground state, shedding new light on the extent of effects of initial retinal configuration on photochemical dynamics of RPs and challenging some of the current mechanisms used to rationalize them. Their additional importance is by offering new archetypes for testing our understanding of RP dynamics and enriching the discourse and tightening control of simulations methods used for this intriguing family of proteins. In particular, since current data present shortening of lifetimes by an order of magnitude (for the ASR) within the same protein, they provide theoreticians with two excellent test cases, promising that such comparison will be significant even at a semi-quantitative level and will not suffer from "oranges and apples" aspects.

\footnotetext{
1. J.L. Spudich, C.-S. Yang, K.-H. Jung, E.N. Spudich, Annu. Rev. Cell Dev. Biol. 16, 365-392 (2000).

2. D. Polli et al., Nature 467, 440-443 (2010).

3. A. Wand, N. Friedman, M. Sheves, S. Ruhman, J. Phys. Chem. B, DOI: 10.1021/jp2125284 (2012).

4. A. Wand, R. Rozin, T. Eliash, K. -H. Jung, M. Sheves, S. Ruhman, J. Am. Chem. Soc. 133, 20922-20932 (2011).

5. O. Bismuth, P. Komm, T. Eliash, N. Friedman, M. Sheves, S. Ruhman, J. Phys. Chem. B 114, 3046-3051 (2010).

6. J.W. Petrich, J. Breton, J.L. Martin, A. Antonetti, Chem. Phys. Lett. 137, 369-375 (1987).

7. A. Kawanabe, H. Kandori, Sensors 9, 9741-9804 (2009).

8. A. Strambi, B. Durbeej, N. Ferré, M. Olivucci, Proc. Natl. Acad. Sci. U.S.A. 107, 21322-21326 (2010).

9. M. Olivucci, A. Lami, F. Santoro, Angew. Chem. Int. Ed. 44, 5118-5121 (2005).

10. A. Sinicropi et al., Proc. Natl. Acad. Sci. U.S.A. 105, 17642-17647 (2008).
} 